\title{
Gastric foveolar-type adenomas endoscopically showing a raspberry-like appearance in the Helicobacter pylori-uninfected stomach
}

\section{다)(i) $\odot=$}

\author{
Authors \\ Kotaro Shibagaki ${ }^{1}$, Chika Fukuyama ${ }^{1}$, Hironobu Mikami ${ }^{1}$, Daisuke Izumi ${ }^{1}$, Noritsugu Yamashita ${ }^{1}$, Tsuyoshi Mishiro', \\ Naoki Oshima', Norihisa Ishimura', Shuichi Sato', Shunji Ishihara', Mamiko Nagase², Asuka Araki², Noriyoshi \\ Ishikawa², Riruke Maruyama², Ryoji Kushima³, Yoshikazu Kinoshita' ${ }^{1}$
}

Institutions

1 Department of Gastroenterology, Faculty of Medicine, Shimane University, Izumo, Japan

2 Department of Pathology, Faculty of Medicine, Shimane University, Izumo, Japan

3 Department of Pathology, Shiga University of Medical Science, Otsu, Japan

submitted 22.8.2018

accepted after revision $\quad 13.12 .2018$

Bibliography

DOI https://doi.org/10.1055/a-0854-3818 |

Endoscopy International Open 2019; 07: E784-E791

(c) Georg Thieme Verlag KG Stuttgart · New York

eISSN 2196-9736

Corresponding author

Kotaro Shibagaki, MD, PhD, Department of

Gastroenterology, Faculty of Medicine, Shimane University, 89-1 Enya, Izumo, Japan 693-8501

Fax: $+81-85320-2187$

kotaro@med.shimane-u.ac.jp

\section{ABSTRACT}

Background and study aims Foveolar-type adenoma is described as a very rare tumor that occurs in individuals without Helicobacter pylori (HP) infection and diagnosed as adenocarcinoma in the Japanese Classification of Gastric Carcinoma (JCGC). However, we have frequently encount- ered patients with foveolar-type adenoma that endoscopically resembles a hyperplastic polyp, suggesting that it has just been overlooked to date. Here, we analyzed clinicopathological characteristics of a special subtype of foveolartype adenoma showing specific endoscopic findings.

Patients and methods From a total of 212 patients with gastric cancer resected during a 22-month period, we enrolled $14(6.6 \%)$ diagnosed with foveolar-type adenoma (adenocarcinoma in JCGC). HP infection status was determined by eradication history, HP serum IgG antibody level, urea breath test, and endoscopic and histological findings. All lesions were observed using white-light endoscopy and narrow-band imaging with magnification endoscopy (NBIME). Endoscopically resected lesions were histologically examined.

Results None of 14 patients had a current or past history of $\mathrm{HP}$ infection. All lesions were visualized on non-atrophic gastric mucosa as small reddish protrusions with fine granular surface, showing a raspberry-like appearance. NBIME showed papillary or gyrus-like microstructures with irregular capillary. Lesions were histologically diagnosed as foveolar-type adenoma showing MUC5AC-positive gastric mucin phenotype. Ki-67 was overexpressed (median labeling index $69.9 \%$, range $28.4-92.1 \%$ ), though all lesions were an intraepithelial tumor without stromal invasion. p53 overstaining was not seen in any.

Conclusions Raspberry-like lesions on non-atrophic gastric mucosa in HP-uninfected individuals should be evaluated for the possibility of a special subtype of foveolar-type adenoma.

\section{Introduction}

Persistent infection of the stomach with Helicobacter pylori (HP) leads to chronic atrophic gastritis and eventually causes gastric cancer $[1,2]$. In Japan, fewer than $1 \%$ of gastric cancers occur in patients not infected by HP $[3,4]$, the majority of which are signet-ring cell carcinomas [5].
Among populations at low risk for gastric cancer, including individuals living in the United States, Nigeria, and Bangladesh, foveolar-type adenoma occurs very infrequently, and is identified as a whitish flat lesion on non-pathologic gastric mucosa [6]. This lesion is histologically characterized by predominant expression of a gastric mucin phenotype with MUC5AC and usually shows high-grade atypical morphological findings [6, 
7]. Western criteria have described such histologic characteristics as foveolar-type adenoma, whereas the diagnosis based on the Japanese Classification of Gastric Carcinoma (JCGC) is welldifferentiated adenocarcinoma [8,9].

Within a relatively short period of time, we have encountered 14 Japanese HP-uninfected patients with a total of 20 foveolar-type adenomas. Their lesions showed the similar macroscopic findings to the hyperplastic polyps that sometimes accompany HP-associated chronic gastritis, and they are quite different from those of traditional foveolar-type adenoma. As far as we could determine by searching the literature, gastric foveolar-type adenoma showing such macroscopic characteristics has never been reported, suggesting that it has been overlooked to date. We carefully analyzed clinical, endoscopic, and histological features of this special subtype of foveolar-type adenoma in the current study. We believe this study will have an influence on existing knowledge about gastric polyps in individuals who are not infected with Helicobacter pylori (HP).

\section{Patients and methods}

\section{Participants}

Between February 2016 and November 2017, a total of 212 patients with gastric cancers were treated by endoscopic or surgical resection at Shimane University Hospital. Among those, 14 (6.6\%) with a total of 20 gastric foveolar-type adenoma polyps (carcinoma in JCGC) were enrolled in this retrospective observational analysis.

The study protocol was approved by the medical ethics committee of Shimane University Hospital and written informed consent was obtained from all participants. Although the study was retrospective, the consecutively enrolled participants faithfully followed the examination protocol. They all underwent history-taking with predetermined medical questions and were asked about HP infection status and a fasting serum gastrin level was obtained. Their lesions were all endoscopically resected after close endoscopic observation and histologically examined. The details are described below.

\section{History-taking and serum gastrin level}

Information regarding each patient's medical history, proton pump inhibitor (PPI) administration, and other medication history, as well as family history of gastric cancer was obtained. The fasting serum gastrin level (normal range, $37-172 \mathrm{pg} / \mathrm{mL}$ ), which may affect development of gastric foveolar hyperplasia [10], was determined in each patient.

\section{Diagnosis of HP infection status}

HP infection status of the patients was determined from findings of the following investigations: medical history of HP eradication therapy, HP serum IgG antibody level, ${ }^{13} \mathrm{C}$-labeled urea breath test, and endoscopic and histologic procedures, as described below. When all of these investigations were negative, a patient was considered to have no present or previous HP infection.

Patients were first asked if they had received HP eradication therapy for a previous infection. Next, an anti-HP serum IgG an- tibody kit (E-Plate, Eiken Chemical Co., Ltd., Tokyo, Japan; cutoff value, $10 \mathrm{U} / \mathrm{mL}$ ) was used, and an antibody titer $<3 \mathrm{U} / \mathrm{mL}$ was considered to indicate a true negative finding to exclude false negatives and the possibility of previous infection [11, 12]. $A{ }^{13} \mathrm{C}$-labeled urea breath test was performed with breath samples obtained before and 20 minutes after oral administration of $100 \mathrm{mg}$ of ${ }^{13} \mathrm{C}$-urea (UBIT tablet $100 \mathrm{mg}$, Otsuka, Tokushima, Japan). Changes in the carbon isotope ratio of carbon dioxide in breath samples were measured using an infrared spectrophotometer (POCone, Otsuka Electronics, Tokyo, Japan), with the cutoff value set at $2.5 \%$.

Gastric mucosal atrophy was observed using endoscopy, with the Kimura-Takemoto classification system used to assess atrophic degree of gastric mucosa. This system classifies mucosal atrophy as either closed or open type [13]. Closed type denotes atrophic mucosa extending from the antrum but without extending to the cardia, and open type denotes that as continuous between the antrum and cardia. The closed type is subclassified into four grades (C0, C1, C2, C3), with $\mathrm{C} 0-1$ indicating that a patient has no or minimal gastric mucosal atrophy, and the open type into three grades $(01,02,03)$.

Finally, resected specimens were histologically evaluated for chronic gastritis according to the updated Sydney system [14]. When mucosal atrophy, intestinal metaplasia, and HP infection were all negative, and there was no or minimal infiltration of neutrophils and mononuclear cells, the resected mucosa was diagnosed as having a non-atrophic mucosal background without HP infection.

\section{Endoscopic procedure}

Images of areas of gastric neoplasia were obtained with white light endoscopy (WLE) and narrow-band imaging combined with magnification endoscopy (NBIME). All lesions were endoscopically resected. First, each was visualized with WLE to identify location, size, shape, color, surface pattern, and surrounding mucosa, then they were visualized with NBIME to evaluate the superficial microstructure and microvascular pattern. Finally, the lesions were resected by endoscopic mucosal resection (EMR) or completely resected by biopsy. All endoscopic procedures were performed by a single expert (K.S.), who had previous experience with more than 5000 magnification endoscopies, or trainees under supervision of that expert.

\section{Histopathologic analysis}

Resected specimens were embedded in paraffin and mounted on slides, then subjected to hematoxylin and eosin staining and immunohistochemistry. Histologic diagnosis was determined based on discussions by expert pathologists (R.M., N.I., A.A., M.N.) in accordance with the Padova/Vienna classification, World Health organization (WHO) classification, and JCGC [8, 15-17]. In addition, immunohistochemistry was performed to assess gastric mucin (MUC5AC for foveolar cells, MUC6 for mucous neck cells or pyloric gland cells) and intestinal mucin (MUC2 for goblet cells, CD10 for intestinal brush border) phenotype. Neoplastic proliferative activity was determined by calculating the Ki-67 labeling index and p53 protein expression was analyzed as a reference finding for TP53 gene 
mutations. Biomarkers used in this study were investigated using monoclonal antibodies to MUC5AC (Novocastra Laboratories Ltd., Newcastle, UK), MUC6 (Novocastra Laboratories), MUC2 (Novocastra Laboratories), CD10 (Dako, Copenhagen, Denmark), P53 (DO-7, Dako), and Ki-67 (MIB-1, Dako).

\section{Endoscope systems}

Instruments used in this study were magnification video-endoscope systems (GIF-H260Z or GIF-H290Z; Olympus Medical Systems Co, Ltd, Tokyo, Japan) and a standard optical video-endoscope system (EVIS LUCERA ELITE system; Olympus Medical Systems). With each system, one light source projects standard broadband white light or, following insertion of an NBI filter into the light path, narrow-band short wavelength light.

\section{Results}

Twenty gastric foveolar-type adenoma lesions in 14 consecutive patients were completely resected with EMR $(n=17)$ or during a biopsy $(n=3)$. Four lesions were histologically evaluated by biopsy before treatment, but the other 16 lesions were diagnosed based on distinctive endoscopic findings to avoid the unintentional resection by biopsy. Median age of the patients was 54 years ( $39-78$ years) and the male-to-female ratio was 9:5. Clinicopathological features of all patients are summarized in $>$ Table 1.

\section{History and serum gastrin level}

Common fundamental diseases included hypertension, hyperlipidemia, and allergic disease, with three patients affected by each. Commonly prescribed medications included statins in three patients and an angiotensin-II receptor blocker in two patients. None of the patients had a family history of gastric cancer. Median serum gastrin level was $89 \mathrm{pg} / \mathrm{mL}(52-330 \mathrm{pg} / \mathrm{mL})$, with only one patient showing slight hypergastrinemia, who was treated with a PPI.

\section{HP infection status}

None of the patients had a positive medical history for HP eradication treatment. All had a HP serum IgG antibody titer $<3 \mathrm{U} / \mathrm{mL}$ and ${ }^{13} \mathrm{CO}_{2}$ excretion in urea breath test results was $<2.5 \%$. The endoscopic grade of gastric mucosal atrophy was C0 for 13 patients and C1 for one. Background gastric mucosa in all patients was histologically diagnosed as non-atrophic fundic gland tissue without HP infection, atrophy, intestinal metaplasia, or infiltration of neutrophils or mononuclear cells. Consequently, all were diagnosed as HP-uninfected.

\section{Endoscopic features}

All lesions were found on non-atrophic gastric mucosa of the gastric body or fornix, and were solitary in 10 and multiple synchronous in four of the 14 patients (28.6\%) ( $\triangleright$ Fig. 1). WLE images of all lesions in this case series are presented in $\mathbf{F i g .} \mathbf{2}$. Each appeared as a small bright red protrusion with a fine granular surface, which showed a raspberry-like appearance. They closely resembled hyperplastic polyps at first glance, which are commonly recognized in cases of HP-associated chronic gastri-
- Table 1 Clinicopathological features of patients with foveolar-type adenoma.

\begin{tabular}{|c|c|}
\hline \multicolumn{2}{|l|}{ Clinical features of all 14 patients } \\
\hline Age, year (range) & $54(39-78)$ \\
\hline Male-female ratio & $9: 5$ \\
\hline Family history of gastric cancer & None \\
\hline Fundamental disease (no. patients) & $\begin{array}{l}\text { Hypertension (3) } \\
\text { Hyperlipidemia (3) } \\
\text { Allergic disease (3) }\end{array}$ \\
\hline Prescribed medication (no. patients) & Statin (3), ARB (2) \\
\hline Serum gastrin level, $\mathrm{pg} / \mathrm{mL}$ (range) & $89(52-330)$ \\
\hline HP infection status & Uninfected \\
\hline \multicolumn{2}{|l|}{ Endoscopic findings of all 20 lesions } \\
\hline Location (no. lesions) & $\mathrm{U}(7), \mathrm{M}(13), \mathrm{L}(0)$ \\
\hline Size, mm (range) & $3(1-5)$ \\
\hline Growth form (no. patients) & $\begin{array}{l}\text { Single (10) } \\
\text { Multiple (4) }\end{array}$ \\
\hline Mucosal atrophy (no. patients) & C0 (13), C1 (1) \\
\hline Gross morphology & Raspberry-like appearance \\
\hline NBIME findings & $\begin{array}{l}\text { Papillary or gyrus-like } \\
\text { microstructure }\end{array}$ \\
\hline \multicolumn{2}{|l|}{ Histologic findings of all 20 lesions } \\
\hline Microscopic morphology & Tubular or papillary \\
\hline Invasion depth & Noninvasive, intraepithelial \\
\hline Mucin phenotype & Pure gastric type (MUC5AC) \\
\hline Ki-67 labeling index \% (range) & $66.9(28.4-92.1)$ \\
\hline P53 staining & Sporadic paterrn \\
\hline \multicolumn{2}{|c|}{$\begin{array}{l}\text { Values for age, serum gastrin level, lesion size, and Ki-67 labeling index are } \\
\text { shown as the median (range). Mucosal atrophy is shown by Kimura-Take- } \\
\text { moto classification system [13]. HP, Helicobacter pylori; U, upper stomach; } \\
\text { M, middle stomach; L, lower stomach; NBIME; narrow-band imaging with } \\
\text { magnification endoscopy; ARB, Angiotensin-II receptor blocker. }\end{array}$} \\
\hline
\end{tabular}

tis. NBIME images of all lesions are presented in > Fig. 3. Each showed papillary or gyrus-like microstructures of varying size and shape, which were clearly framed by a transparent whitish margin. Capillaries were irregularly dilated with dense growing or obscurely visualized. The surrounding mucosa showed regularly arranged round pits, suggesting non-atrophic fundic gland mucosa. The lesions were clearly distinguishable from surrounding mucosa based on differences in microstructure and capillary patterns of the mucosal surface, thus satisfying endoscopic diagnostic criteria for gastric cancer $[18,19]$.

\section{Histopathologic features}

Representative histopathologic images are presented in - Fig. 4. The resected specimens showed small protrusions with a median size of $3 \mathrm{~mm}(1-5 \mathrm{~mm})$. Neoplastic glands showed tubular or papillary growth with structural atypia, and were clearly demarcated from surrounding mucosa. Neoplastic 


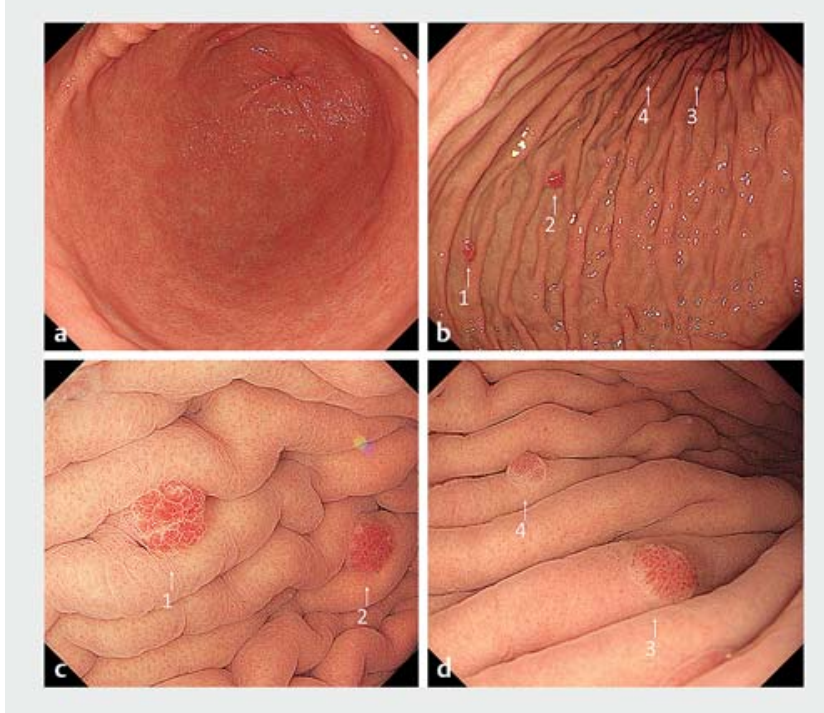

- Fig. 1 Representative case of multiple foveolar-type adenoma. a White-light endoscopy (WLE) findings showed normal gastric mucosa without atrophic change. b A total of 4 small protruding lesions were found at the greater curvature of the gastric body and numbered 1, 2, 3, and 4 for analysis. c, $\mathbf{d}$ Underwater observation provided fine endoscopic images of the lesions, shown as small bright red protrusions with a fine granular surface, which we considered to be a raspberry-like appearance. Endoscopic images of lesions 1, 2, 3, and 4 by WLE and narrow-band imaging with magnification endoscopy are presented as $n 1, n 2, n 3$, and $n 4$ in $\checkmark$ Fig. 2 and $>$ Fig. 3 , respectively.

cell density was highly increased, and neoplastic nuclei were round or cuboidal with loss of polarity. All areas of neoplasia were retained within the intraepithelial layer without stromal or lymphovascular invasion. Background mucosa showed nonatrophic fundic glands without a histologic component fundic gland polyp such as cystically dilated and irregularly deformed oxyntic glands with buds.

Immunohistochemistry findings showed that all lesions were positive for MUC5AC expression and negative for MUC6, MUC2, and CD10 expression, nd thus positive for a pure gastric mucin phenotype MUC5AC. Ki-67 was highly overexpressed with a median Ki-67 labeling index of $69.9 \%$ (28.4-92.1\%). The p53 protein was sporadically stained in all lesions, suggesting the wild-type TP53 gene.

The lesions were diagnosed as foveolar-type adenoma; noninvasive high-grade neoplasia based on the Padova/Vienna classification $[15,16]$, high-grade intraepithelial dysplasia by the WHO classification [17], and well-differentiated tubular or papillary adenocarcinoma using the JCGC [8].

\section{Discussion}

The foveolar-type adenoma lesions in this study were located on non-atrophic gastric fundic gland mucosa in the absence of HP infection and showed a MUC5AC-positive pure gastric mucin phenotype, similar to traditional foveolar-type adenoma. However, macroscopic findings were quite different from those of traditional cases and compared with the entire study population, the proportion with gastric cancer was not especially low. Gastric foveolar-type adenoma with such unique endoscopic findings as were seen in this study has never been reported. Consequently, we consider these two types of foveolar type adenomas as different tumors in terms of molecular biology, although they show similar histologic characteristics.

Gastric fundic gland polyp (FGP)-associated dysplasia/adenocarcinoma could be one of the differential diagnoses, because the neoplastic epithelium shows foveolar differentiation in our cases as well as in fundic gland polyps [17,20]. Gastric FGP reportedly rarely accompanies gastric cancer and has endoscopic and histologic findings quite similar to those in our cases [20]. However, as shown in $>\mathbf{F i g . 4}$, the lesions in our cases did not accompany the histologic components of FGP. In this respect, we plan to perform molecular biological analysis, including beta-catenin (CTNNB1) mutations [21].

There are well-known discrepancies between histologic diagnostic criteria for early gastric cancer developed by Western and Japanese pathologists. Japanese criteria emphasize not only invasion but also cellular atypia, leading to "carcinoma in situ," which is usually diagnosed as adenoma or dysplasia based on Western criteria. In addition, gastric neoplastic lesions with a pure gastric mucin phenotype likely have poorly differentiated components or show lymph node metastasis regardless of degree of atypical morphology [22-26]. Consequently, the current cases were diagnosed with adenocarcinoma based on the JCGC, although they had neither invasive nor metastatic behavior. A dual diagnosis for the same neoplasm is very confusing, but at the moment, we have no choice but to use these two diagnoses separately according to the contents of analysis or discussion.

Gastric cancer without HP infection is now being intensively discussed in Japan, as prevalence of HP infection recently has decreased [27]. According to previous reports, the percentage of patients with gastric cancer who are not infected with HP ranges from $0.42 \%$ to $0.66 \%$ of all with gastric cancer in Japan $[3,4]$. The majority of these cases reportedly have been signetring cell carcinoma $[3-5,28]$, whereas that was not found in the current cohort. On the other hand, individuals with foveolar-type adenoma (adenocarcinoma in JCGC) account for $6.6 \%$ (14/212) of all patients with gastric cancer, suggesting a large discrepancy as compared to the previously reported proportion of HP-unassociated gastric cancers.

Gross morphology of the foveolar-type adenoma lesions in the current study was quite similar to that of hyperplastic polyps that sometimes accompany HP-associated chronic gastritis. In addition, all of the current lesions were small, and $28.6 \%$ (4/14) were multiple synchronous. These endoscopic findings are suggestive of a non-neoplastic nature. Moreover, even when biopsy is performed, histologic differential diagnosis between foveolar-type adenoma and regenerative atypia is reportedly sometimes difficult for pathologists who have little experience with this tumor [29]. We believe that presence of macroscopic similarities to hyperplastic polyps as well as difficulty with histologic differential diagnosis may explain why this neoplasia has been previously overlooked in endoscopic as- 

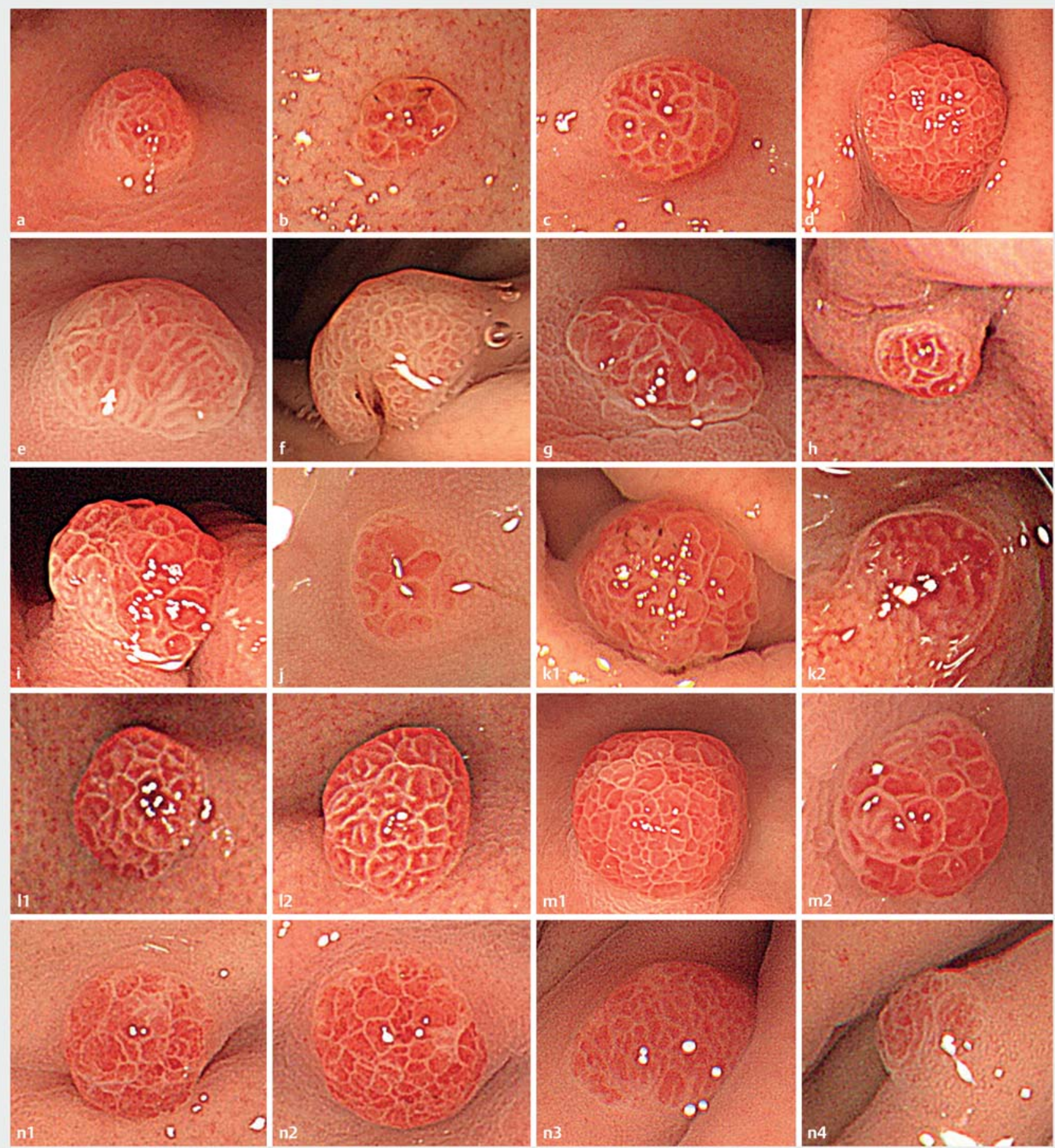

- Fig. 2 White-light endoscopic images of all 20 foveolar-type adenoma lesions detected in the present study. Each was recognized as a bright reddish small protrusion with a fine granular surface, which showed a raspberry-like appearance. The lesions were solitary in 10 patients (a to $\mathbf{j}$ ) and multiple synchronous in 4 (k1-2, $1 \mathbf{1}-\mathbf{2}, \mathbf{m} 1 \mathbf{1}-\mathbf{2}, \mathbf{n} \mathbf{1}-\mathbf{4})$.

sessments. Nevertheless, a hyperplastic polyp-like lesion on non-atrophic gastric mucosa in the absence of HP infection is an unusual endoscopic finding, which led to our initial awareness of this neoplasia. In addition, NBIME findings sufficiently met the endoscopic diagnostic criteria for early gastric cancer
$[18,19]$, suggesting the efficacy of this methodology for endoscopic diagnosis of this neoplastic lesion.

The foveolar-type adenomas in this study showed a pure gastric mucin phenotype and Ki-67 overexpression, both of which usually indicate a high malignant potential and poor outcome [22-25, 30, 31]. However, all of the lesions were discov- 

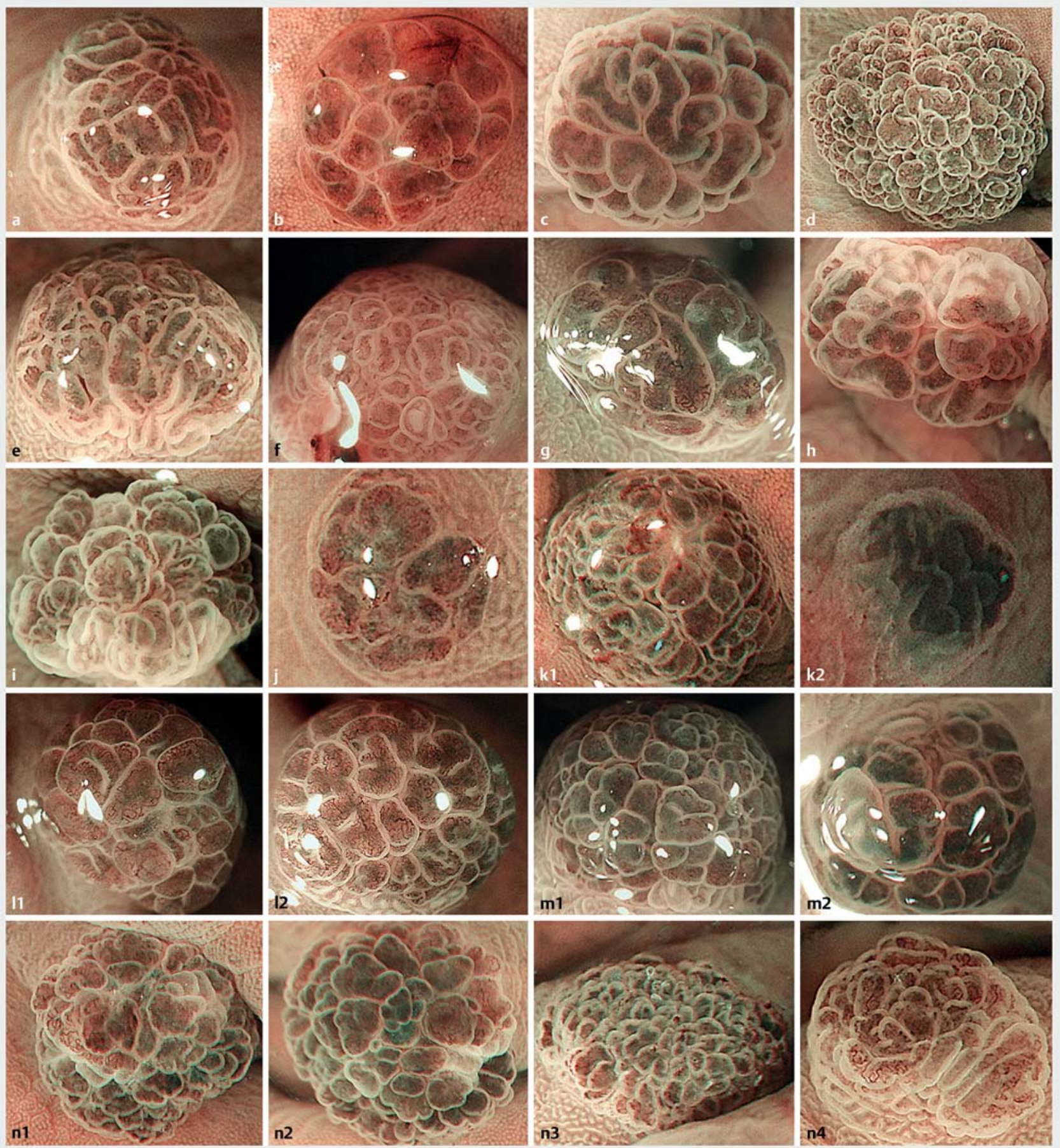

- Fig. 3 Magnification endoscopic images with narrow-band imaging of all 20 foveolar-type adenoma lesions detected in the current study. Each numbered photograph corresponds to the numbers shown in $\mathbf{F i g . 2}$. Neoplastic lesions showed papillary or gyrus-like microstructures of varying sizes and shapes. Capillaries were irregularly dilated with dense growing or sometimes obscurely visualized. Surrounding mucosa had regularly arranged round pits, suggesting non-atrophic fundic gland mucosa.

ered when small, and did not show invasive or metastatic behavior. Advanced disease has never been reported with this type of neoplasia. Therefore, the lesions may be clinically indolent and have a negligible effect on patient prognosis even without treatment, although that speculation contradicts histologic diagnosis of adenocarcinoma according to the JCGC.

Chronic mucosal inflammation caused by HP infection might be necessary for gastric neoplasia lesions to spread, and manifest their invasive and metastatic behaviors. Persistent HP in- 

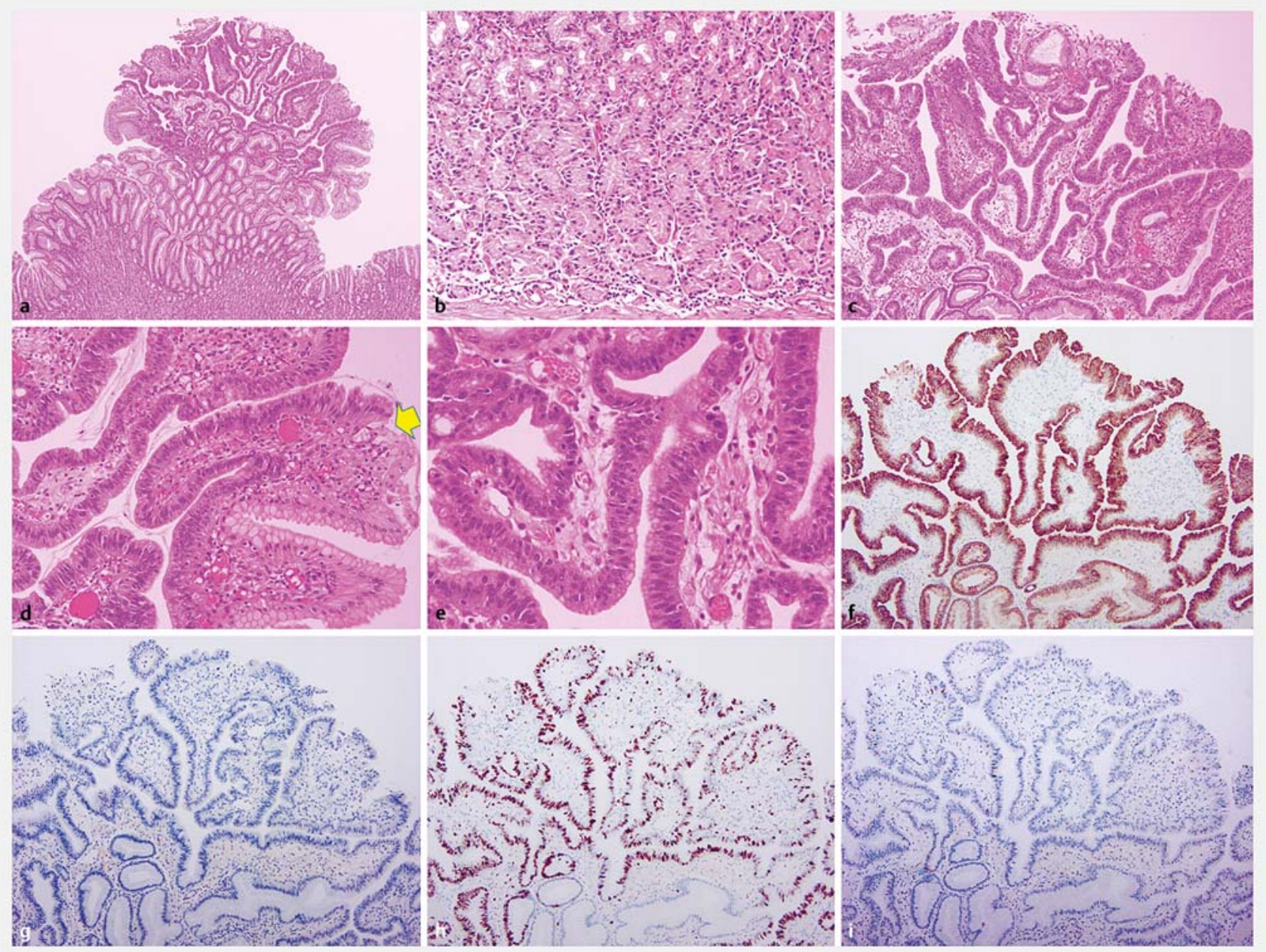

\begin{abstract}
- Fig. 4 Histopathologic features of multiple foveolar-type adenoma lesions in the same patient shown in $>$ Fig. 1. a The specimen labelled lesion 1 in $>$ Fig. 1 showed a protruded tumor $\mathbf{b}$ on a non-atrophic normal fundic gland without cystically dilated and irregularly deformed oxyntic glands. c Neoplastic glands had severe structural atypia and $\mathbf{d}$ were clearly demarcated from the surrounding mucosa (yellow arrow). e Neoplastic nuclei were rounded or cuboidal, with loss of polarity. $\mathbf{f}$ Immunohistochemistry showed that neoplastic cells were positive for MUC5AC expression and $\mathbf{g}$ negative for MUC6, MUC2, and CD10 expression. h Ki-67 was overexpressed (labeling index $92.1 \%$ ). $\mathbf{i}$ The p53 protein was only sporadically stained, suggesting the wild-type TP53 gene.
\end{abstract}

fection leads to a variety of epigenetic modifications, resulting in gastric carcinogenesis, mainly via silencing of tumor suppressor genes $[32,33]$. Signet-ring cell carcinomas in HP-uninfected cases reportedly show lower proliferative capacity, less extensive spreading, and slower progression as compared to those in HP-infected cases [28]. However, tumorigenic mechanisms of gastric cancers in an HP-uninfected population have not yet been investigated, except for hereditary diffuse gastric cancer, which occurs regardless of HP infection based on germline mutations of $C D H 1$ (epithelial cadherin gene) [34]. None of the patients in the current study had an HP infection or family history of gastric cancer. In addition, p53 protein over-staining was not seen in any of the lesions, suggesting that this neoplasia occurs independent of multistep tumorigenesis, unlike HPassociated gastric cancers [35]. Other factors for gastric mucosa proliferation include hypergastrinemia, which reportedly has effects on hyperplastic growth of gastric foveolar epithelium
[10]. However, the serum gastrin levels in the current patients were within normal range, except for one who was administered a PPI. Consequently, we consider that tumor a result of a sporadic somatic mutation and unrelated to chronic inflammation, hereditary predisposition, or other sequential disease.

This study has some limitations. First, it was a retrospective observational analysis of an unselected but small population. A multicenter prospective investigation of a larger population is necessary to determine the exact incidence rate of foveolartype adenoma in HP-uninfected individuals. Second, only morphological analysis was done. Molecular biological analysis is necessary to reveal not only the causal genetic mutation of this special subtype of foveolar-type adenoma but also the essential difference between it and traditional foveolar-type adenoma. We are now performing whole-exome sequencing of the current neoplastic lesions to reveal any related causal genetic mutation. 


\section{Conclusion}

In conclusion, a special subtype of foveolar-type adenoma may just have been overlooked as a non-neoplastic lesion. When a raspberry-like lesion is found in the stomach of an HP-uninfected individual, the possibility that the lesion may be neoplastic should be considered.

\section{Competing interests}

None

\section{References}

[1] Kusters JG, van Vliet AH, Kuipers EJ. Pathogenesis of Helicobacter pylori infection. Clin Microbiol Rev 2006; 19: 449-490

[2] Helicobacter and Cancer Collaborative Group. Gastric cancer and Helicobacter pylori: A combined analysis of 12 case control studies nested within prospective cohorts. Gut 2001; 49: 347-353

[3] Matsuo T, Ito M, Takata S et al. Low prevalence of Helicobacter pylorinegative gastric cancer among Japanese. Helicobacter 2001; 16 : $415-419$

[4] Ono S, Kato M, Suzuki M et al. Frequency of Helicobacter pylori-negative gastric cancer and gastric mucosal atrophy in a Japanese endoscopic submucosal dissection series including histological, endoscopic and serological Atrophy. Digestion 2012; 86: 59-65

[5] Kiso M, Yoshihara M, Ito $\mathrm{M}$ et al. Characteristics of gastric cancer in negative test of serum anti-Helicobacter pylori antibody and pepsinogen test: a multicenter study. Gastric Cancer 2017; 20: 764 - 771

[6] Fenoglio-Preiser's Gastrointestinal Pathology. 4th. Amy EN (Ed). Wolters Kluwer; 2017

[7] Park DY, Srivastava A, Kim GH et al. Adenomatous and foveolar gastric dysplasia: distinct patterns of mucin expression and background intestinal metaplasia. Am J Surg Pathol 2008; 32: 524-533

[8] Japanese Gastric Cancer Association. Japanese gastric cancer treatment guideline 2010: 3rd English edition. Gastric Cancer 2011; 14: $113-123$

[9] Sekine S, Yoshida $\mathrm{H}$, Jansen $\mathrm{M}$ et al. The Japanese viewpoint on the histopathology of early gastric cancer. Adv Exp Med Biol 2016; 908: $331-346$

[10] Hongo M, Fujimoto K. Gastric Polyps Study Group. Incidence and risk factor of fundic gland polyp and hyperplastic polyp in long-term proton pump inhibitor therapy: a prospective study in Japan. J Gastroenterol 2010; 45: 618-624

[11] Ootani H, Iwakiri R, Shimoda R et al. Role of Helicobacter pylori infection and nonsteroidal anti-inflammatory drug use in bleeding peptic ulcers in Japan. J Gastroenterol 2006; 41: 41-46

[12] Kishikawa H, Kimura K, Takarabe S et al. Helicobacter pylori antibody titer and gastric cancer screening. Dis Markers 2015; 2015: 156719

[13] Kimura K, Takemoto T. An endoscopic recognition of atrophic border and significance in chronic gastritis. Endoscopy 1969; 3: 87-97

[14] Dixon MF, Genta RM, Yardley JH et al. Classification and grading of gastritis. The updated Sydney System. International Workshop on the Histopathology of Gastritis, Houston 1994. Am J Surg Pathol 1996; 20: $1161-1181$

[15] Rugge M, Correa P, Dixon MF et al. Gastric dysplasia: the Padova international classification. Am J Surg Pathol 2000; 24: 167-176
[16] Schlemper R], Riddell RH, Kato Y et al. The Vienna classification of gastrointestinal epithelial neoplasia. Gut 2000; 47: 251-255

[17] Lauwers G, Carneiro F, Graham D et al. WHO classification of tumors of the digestive system. 4th ed. Lyon: JARC; 2010

[18] Eozoe $\mathrm{Y}$, Muto $\mathrm{M}$, Uedo $\mathrm{N}$ et al. Magnifying narrowband imaging is more accurate than conventional white-light imaging in diagnosis of gastric mucosal cancer. Gastroenterology 2011; 141: 2017-2025

[19] Yao K, Anagnostopoulos GK, Ragunath K. Magnifying endoscopy for diagnosing and delineating early gastric cancer. Endoscopy 2009; 41 $462-467$

[20] Togo K, Ueo T, Yonemasu $\mathrm{H}$ et al. Two cases of adenocarcinoma occurring in sporadic fundic gland polyps observed by magnifying endoscopy with narrow band imaging. World J Gastroenterol 2016; 22: $9028-9034$

[21] Abraham SC, Nobukawa B, Giardiello FM et al. Sporadic fundic gland polyps: common gastric polyps arising through activating mutations in the beta-catenin gene. Am J Pathol 2001; 158: 1005-1010

[22] Saito A, Shimoda T, Nakanishi Y et al. Histologic heterogeneity and mucin phenotypic expression in early gastric cancer. Pathol Int 2001; 51: $165-171$

[23] Kabashima A, Yao T, Sugimachi K et al. Relationship between biologic behavior and phenotypic expression in intramucosal gastric carcinomas. Hum Pathol 2002; 33: 80-86

[24] Koseki K, Takizawa T, Koike M et al. Distinction of differentiated type early gastric carcinoma with gastric type mucin expression. Cancer 2000; 89: $724-732$

[25] Tajima Y, Yamazaki K, Makino R et al. Gastric and intestinal phenotypic marker expression in early differentiated-type tumors of the stomach: clinicopathologic significance and genetic background. Clin Cancer Res 2006; 12: 6469-6479

[26] Ueyama H, Matsumoto K, Nagahara A et al. Gastric adenocarcinoma of the fundic gland type (chief cell predominant type). Endoscopy 2014; 46: $153-157$

[27] Inoue M. Changing epidemiology of Helicobacter pylori in Japan. Gastric Cancer 2017; 20: 3 -7

[28] Horiuchi Y, Fujisaki J, Yamamoto $\mathrm{N}$ et al. Biological behavior of the intramucosal Helicobacter pylori-negative undifferentiated-type early gastric cancer: comparison with Helicobacter pylori-positive early gastric cancer. Gastric Cancer 2016; 19: 160 - 165

[29] Serra S, Ali R, Bateman AC et al. Gastric foveolar dysplasia: a survey of reporting habits and diagnostic criteria. Pathology 2017; 49: 391 396

[30] Luo G, Hu Y, Zhang Z et al. Clinicopathologic significance and prognostic value of Ki-67 expression in patients with gastric cancer: a meta-analysis. Oncotarget 2017; 8: $50273-50283$

[31] Ko GH, Go SI, Lee WS et al. Prognostic impact of Ki-67 in patients with gastric cancer-the importance of depth of invasion and histologic differentiation. Medicine 2017; 96: e7181

[32] Maekita T, Nakazawa K, Mihara M et al. High levels of aberrant DNA methylation in Helicobacter pylori-infected gastric mucosae and its possible association with gastric cancer risk. Clin Cancer Res 2006; 12: 989-995

[33] Niwa T, Tsukamoto T, Toyoda T et al. Inflammatory processes triggered by Helicobacter pylori infection cause aberrant DNA methylation in gastric epithelial cells. Cancer Res 2010; 70: 1430 - 1440

[34] Hansford S, Kaurah P, Li-Chang $\mathrm{H}$ et al. Hereditary diffuse gastric cancer syndrome: $\mathrm{CDH} 1$ mutations and beyond. JAMA Oncol 2015; 1 : $23-32$

[35] Wei ], Nagy TA, Vilgelm A et al. Regulation of p53 tumor suppressor by Helicobacter pylori in gastric epithelial cells. Gastroenterology 2010: 139: $1333-1343$ 